\title{
Spatiotemporal Characterization of Sclerotinia Crown Rot Epidemics in Pyrethrum
}

Jason B. Scott, Tasmanian Institute of Agriculture (TIA), University of Tasmania-Cradle Coast Campus, Burnie, Tasmania, 7320, Australia; David H. Gent, United States Department of Agriculture-Agricultural Research Service, Forage Seed and Cereal Research Unit, Oregon State University, Department of Botany and Plant Pathology, Corvallis 97331; Sarah J. Pethybridge, The New Zealand Institute of Plant \& Food Research Limited, Private Bag 4704, Lincoln, Christchurch, New Zealand; and Frank S. Hay, TIA, University of Tasmania-Cradle Coast Campus, Tasmania

\begin{abstract}
Scott, J. B., Gent, D. H., Pethybridge, S. J., and Hay, F. S. 2014. Spatiotemporal characterization of Sclerotinia crown rot epidemics in pyrethrum. Plant Dis. 98:267-274.

Sclerotinia crown rot, caused by Sclerotinia minor and S. sclerotiorum, is a disease of pyrethrum in Australia that may cause substantial decline in plant density. The spatiotemporal characteristics of the disease were quantified in 14 fields during three growing seasons. Fitting the binary power law to disease incidence provided slope $(b=1.063)$ and intercept $\left(\ln \left(A_{p}\right)=0.669\right)$ estimates significantly $(P \leq 0.0001)$ greater than 1 and 0 , respectively, indicating spatial aggregation at the sampling unit scale that was dependent upon disease incidence. Covariate analyses indicated that application of fungicides did not significantly influence these estimates. Spatial autocorrelation and spatial analysis by distance indices indicated that spatial aggregation above the sampling unit scale was limited to 20 and $17 \%$ of transects analyzed, respectively. The range of significant aggregation was limited primarily to neighboring sampling units only. Simple temporal disease models

failed to adequately describe disease progress, due to a decline in disease incidence in spring. The relationships between disease incidence at the scales of individual plants within quadrats and quadrats within a field was modeled using four predictors of sample size. The choice of the specific incidence-incidence relationship influenced the classification of disease incidence as greater than or less than $2 \%$ of plants, a provisional commercial threshold for fungicide application. Together, these studies indicated that epidemics of Sclerotinia crown rot were dominated by small-scale aggregation of disease. Larger scale patterns of diseased plants, when present, were associated with severe disease outbreaks. The spatial and temporal analyses were suggestive of disease epidemics being associated with localized primary inoculum and other factors that favor disease development at a small scale.
\end{abstract}

The herbaceous perennial crop pyrethrum (Tanacetum cinerariifolium (Trevir.) Sch. Bip.) is grown for the production of the insecticidal pyrethrin compounds, produced in the oil glands of the achenes within flowers $(5,30)$. Australia currently supplies approximately $60 \%$ of the world's pyrethins. Globally, pyrethrum is typically grown as a low-input subsistence crop. In contrast, Australian production is a highly intensive system and yields are responsive to high inputs of agrichemicals for weed and disease management (21). Ideally, production is perennial, with three or more flower harvests occurring at approximately 15 months post sowing and annually thereafter.

Early termination and sowing an alternative crop in the rotation prior to the ideal scenario of three of more pyrethrum harvests is often associated with high disease pressure. Among the diseases of known importance in pyrethrum production is Sclerotinia crown rot (SCR). SCR is caused by the fungal pathogens Sclerotinia minor and $S$. sclerotiorum, of which $S$. minor is dominant (21). The disease most frequently occurs from austral mid-autumn (April) to austral mid-spring (October). Symptoms of SCR include wilting and early senescence of leaves. Cottony, mycelial growth and the formation of sclerotia on diseased plants are also commonly observed (21). Loss of crop canopy as a result of leaf senescence and

Corresponding author: J. B. Scott, E-mail: Jason.Scott@utas.edu.au

Mention of a trademark, proprietary product, or vendor does not constitute a guarantee or warranty of the product by the USDA and does not imply its approval to the exclusion of the products or vendors that may also be suitable.

Accepted for publication 10 September 2013.

http://dx.doi.org/10.1094/PDIS-07-13-0692-RE

(C) 2014 The American Phytopathological Society plant death reduces stand density and allows for increased competition from weeds. Additionally, sclerotia may serve as a source of inoculum for Sclerotinia flower blight of pyrethrum and for other susceptible crop species within the rotation $(13,21)$. However, the strength of this association has yet to be determined.

Sclerotinia spp. survive as melanized sclerotia, which can remain viable for more than 8 years in the soil environment (1). Myceliogenic germination of sclerotia is thought to be the principle mechanism by which the pathogen infects and causes SCR in pyrethrum (21). Avoidance of fields infested with S. minor or S. sclerotiorum sclerotia is largely impractical, due to the broad host range of these pathogens, which include potato, carrot, and bean crops which are typically used in rotation with pyrethrum $(2,12)$.

Management of SCR in pyrethrum is reliant upon the application of the dicarboximide fungicide procymidone. Fields with a high disease incidence may receive up to three applications over autumn and winter prior to first harvest. Timing of the first application is often based on a provisional action threshold of $2 \%$ disease incidence. However, economic justification for this threshold has not been modeled. Subsequent applications at 3- to 4-week intervals may be recommended, dependent upon disease levels and environmental conditions.

The primary objective of this study was to provide quantitative data on the spatiotemporal attributes of SCR epidemics in pyrethrum. The theory that observed variation in these variables would differ under uncontrolled and disease-managed conditions was explored. Additionally, the implications for disease incidence assessment were examined.

\section{Materials and Methods}

Fields and data collection. Model construction data set. SCR incidence was assessed in 14 commercial pyrethrum fields over three cropping seasons-2010 (five fields), 2011 (five fields), and 2012 (four fields) - in northwest Tasmania, Australia. All fields 
were planted the preceding austral spring (September 2009, 2010, and 2011, respectively) and were assessed prior to their first harvest in January 2011, 2012, and 2013, respectively. Within each field, two plot areas were marked for assessment. The first plot (nontreated) did not receive applications of any fungicides with efficacy for the control of Sclerotinia spp. during the assessment period. The second plot, situated parallel to the nontreated plot, received the standard commercial control practice for that field. These plots received between one and three applications of the fungicide procymidone (Fortress 500; Crop Care Australasia Pty. Ltd.) at the industry recommended rate (1.6 liter/ha) during the assessment period. All plots were $30 \mathrm{~m}$ long and between 18 and $24 \mathrm{~m}$ wide, dependent upon the spray boom width employed for a given field. Six assessments of each plot were undertaken from austral mid-autumn (April to May) to austral mid-spring (September to October) each year. Intervals between sampling periods varied, with a mean interval of 27.3 days (range of 13 to 41 days).

Disease incidence was assessed using a $1-\mathrm{m}^{2}$ grid (sampling unit) consisting of 25 individual squares, each 20 by $20 \mathrm{~cm}$, in a five-by-five arrangement. Due to irregular growth habits of individual plants and highly variable population densities, differentiating individual pyrethrum plants is often impractical. Thus, each square within a sampling unit was treated as an analog for a single pyrethrum plant and, for simplicity, is referred to as such hereafter. At each sampling time point, disease was measured within each plot along two 25-m-long transects, with sampling units at each 1-m interval $(N=25)$. Within each sampling unit, the number of plants $(n)$ and number of diseased plants exhibiting visual symptoms of SCR $(x)$ were counted. As such, $n$ was variable between sampling units, with a mean across all data of 20.6, standard deviation of 4.3, and a range from 1 to 25 . Symptomatic SCR plants were characterized by the presence of white, cottony mycelia growth, or sclerotia on wilted tissue. The estimated mean incidence, expressed as a proportion, of diseased plants $\hat{p}$ was calculated as $\hat{p}=\Sigma x_{i} / \Sigma n_{i}$, where $x_{i}$ is the number of diseased plants and $n_{i}$ is the number of plants present in the $i$ th sampling unit.

Model validation data set. An additional 72 commercial fields were assessed at three times during 2010 (24 fields), 2011 (22 fields), and 2012 (26 fields). Data were used for validation of relationships between disease incidence in spatial hierarchies, as described below. These fields were dispersed across all production regions in northern Tasmania, encompassing an area approximately $260 \mathrm{~km}$ in diameter. Field design and treatments were the same as for the model construction dataset, with the exception that plots were $10 \mathrm{~m}$ in length. Individual plots were assessed using the same sampling unit design, with 25 randomly selected sampling units assessed for disease. Disease assessment times were equivalent to the first, third, and fifth sampling times described above.

Temporal analysis. Disease progress over time was modeled for each plot within each field in the model construction data (16). Linearized forms of the linear, monomolecular, exponential, logistic, and Gompertz models were fit to mean disease incidence at the plot scale (i.e., mean of all 25 sampling units) across all sampling periods using ordinary least-squares linear regression. Goodness-of-fit statistics were examined to determine the predictive power of each model for an individual plot. To compare their relative fit, fitted values from each model were back-transformed, and the back-transformed coefficient of variation $\left(R^{* 2}\right)$ calculated. The model of best fit was determined, in order of preference, using the $F$ statistics for linearity, the coefficient of determination $\left(R^{* 2}\right)$, the residual standard error, and visual comparisons of the plot of residuals against predicted values $(16,17)$. Model fitting were conducted in $R$ v. 2.15 .1 (24).

Binary power law. Model construction data set. The binary form of Taylor's power law expresses the relationship between the observed variance of disease incidence and the theoretical variance of a binomial distribution (9). Agreement with a binomial distribution is considered indicative of a random spatial pattern of disease incidence at the scale of the sampling unit and below. This relationship can be used to characterize overall spatial aggregation of dis- ease incidence at varying spatial scales and time (15). The linearized form of the model after natural log-transformation is:

$$
\ln \left(v_{o b s}\right)=\ln \left(A_{p}\right)+b \ln \left(v_{b i n}\right)
$$

where the observed variance is estimated by $v_{o b s}=\left[\sum n_{i}^{2}\left(p_{i}-\right.\right.$ $\left.\hat{p})^{2}\right] /\left[\bar{n}^{2}(N-1)\right]$, the binomial variance by $v_{\text {bin }}=\hat{p}(1-\hat{p})^{2} / \bar{n}$, and incidence in the $i$ th sampling unit as $p_{i}=x_{i} / n_{i}$ (15). The parameters $x, n$, and $N$ are as defined previously, and $\bar{n}$ is the mean of $n$ for a given data set. When $A_{p}=1$ and $b=1$, equation 1 indicates that the disease distribution is adequately described by the binomial distribution, and thus a random spatial pattern. When $A_{p}>1$ and $b=1$, disease incidence has an aggregated spatial pattern, where the degree of aggregation is not dependent upon $p$; values of $b>1$ indicate that aggregation is systematically related to $p$. Estimates of $v_{\text {obs }}$ and $v_{\text {bin }}$ were calculated at two spatial scales, plots within a field (two plots per field) and transects within plots (two transects per plot), for all fields at each sampling period where the total number of diseased plants observed was greater than 1 . Ordinary least-squares regression was used to estimate $A_{p}$ and $b$ using $R$. Deviations of $A_{p}$ and $b$ from 1 were assessed using $t$ tests. Additionally, slope and intercept parameter estimates at each spatial scale were compared by including estimates obtained at the plot scale as terms within a model describing the binary power law at the transect scale. The plot-scale slope parameter was included as a slope offset term, while the plot-scale intercept parameter was subtracted from the response variable. The slope and intercept parameters of this model were tested individually for deviation from 0 with $t$ tests, which would indicate significant difference in parameter estimates between spatial scales.

To assess the influence of year (Year), plot treatment (Treatment), site (Site), and sampling period (Sample period) on the estimates of $A_{p}$ and $b$, a covariate analysis was conducted within $R$. The model defined by equation 1 was considered the null model. Factors were separately included, first as an intercept term, then as an interaction term with the slope. The factor Year was included as a covariate term within the model across the complete data set, whereas Treatment, Site, and Sample period were included as terms within individual years only. A factor was considered significant if its inclusion reduced the sum of squares error (SSE) of this model significantly $(P<0.05)$ relative to the null model. Significance of the difference in SSE between models was calculated by an $F$ test, where $F=\left(\right.$ factor SSE $\left./ d f_{\text {factor }}\right) /\left(\right.$ model SSE $\left./ d f_{S S E}\right)$ and $d f_{\text {factor }}$ and $d f_{S S E}$ are the degrees of freedom of the factor and model sum of squares, respectively.

Model validation data set. Estimates of disease incidence obtained from the validation data set were modeled using the binary power law, as described previously. The estimated parameters were assessed for deviation from previous values by including this plotscale slope parameter as an offset term, while the plot-scale intercept was subtracted from all response variables. The residual intercept and slope parameters in this second model were then tested for differences from 0 using $t$ tests.

Correlation-based spatial analyses. Spatial autocorrelation. For each transect where the number of sampling units containing one or more diseased plants was greater than one, within each sampling period, spatial autocorrelation coefficients were calculated. Prior to the calculation of correlation coefficients, the Haldane transformation $[Y=\ln (y /[1-y])]$ was performed on the data, where $y=(x+0.5) /(n+1)$ and $x$ and $n$ are as defined previously. This correction was employed to avoid taking a logarithm of, or divide by, $0(25,26)$. Spatial autocorrelation coefficients were calculated by (15):

$$
\hat{r}(j)=\hat{C}(j) / \hat{C}(0)
$$

where $\hat{C}(0)$ is the variance defined by $\hat{C}(0)=\Sigma\left(Y_{i}-\bar{Y}\right)^{2} / N$, and $Y_{i}$ is the value of $Y$ at the $i$ th sampling unit, $\bar{Y}$ is the mean of $Y$ across the transect and $N$ is the number of sampling units. The autocovariance between values of $Y_{i}$ at a spatial lag of $j$ defined by $\hat{C}(j)$ $=\Sigma\left[\left(Y_{i}-\bar{Y}\right)\left(Y_{i+j}-\bar{Y}\right) / N_{j}\right]$, where $N_{j}$ is the number of pairs of sampling units separated by a spatial lag of $j$. Significance was deter- 
mined by constructing 1,000 random permutations for each transect and comparing the estimated coefficient to the distribution of permutation coefficients. Calculations were performed in $R$.

Spatial analysis by distance indices. Spatial analysis by distance indices (SADIE) (18) was conducted using counts of the number of diseased plants per sampling unit. All transects where the observed number of sampling units containing at least one diseased plant was greater than 1 were analyzed. SADIE uses the concept of distance to regularity, the number of "moves" individuals (in this study, diseased plants) must make to produce a completely regular distribution across all sampling units $(18,19)$. The significance of the estimated distance to regularity $\left(D_{r}\right)$ is determined by conducting a series of random reassignments of counts within the sampled area and comparing $D_{r}$ with the distribution of estimates obtained from the randomizations. An index of aggregation is obtained using the formula:

$$
I_{a}=D_{r} / E_{a}
$$

where $E_{a}$ is the average distance to regularity obtained from randomization. Values of 1 indicate a random distribution, while values greater than 1 are indicative of spatial aggregation. An $I_{a}$ value less than 1 indicates a regular spatial pattern. For each transect, the maximum number of randomizations available within SADIE $(n=$ $5,967)$ was conducted for each analysis.

The SADIE transportation algorithm also attributes to each sampling unit within a transect the standardized indices indicating net inflow $\left(v_{j}\right)$ and outflow $\left(v_{i}\right)$ of individuals required to achieve regularity (20). By accounting for the spatial location of sampling units, these indices can be used to define and measure the size of clusters of individuals. Spatial areas of high outflows (i.e., the number of individuals present are greater than average across all sampling units) are termed "red" clusters in this system, while clusters of high inflows (i.e., fewer individuals than average), are termed "blue" clusters (20). For all transects where $I_{a}$ was significantly greater than $1(P \leq 0.025$, under a two-tailed test), the number and size, in sampling units, of each red cluster of diseased plants was determined using the arbitrary boundary definition of $v_{i}=1.5$, as outlined by Perry et al. (20).

Relationships between disease incidence at the sampling unit and plant scales. The relationship of disease incidence at different spatial scales was examined by comparing observed disease incidence at the plant scale $(\hat{p})$ to that at the sampling unit scale $\left(p_{s u}\right)$ within individual plots. If $p$ can be described by a binomial distribution, then (14):

$$
\tilde{p}_{s u}=1-(1-\hat{p})^{n}
$$

where $n$ represents the number of plants within a sampling unit; in this study, where $n$ was variable, $n$ was approximated by the mean of $n$ across all plots, sites, and sampling units $(\bar{n})$. The tilde $(\sim)$ indicates that the estimate of $p_{s u}$ is derived from measures at a different spatial scale. To account for spatial heterogeneity, $n$ can be replaced with an estimate of "effective sample size". The method of Rao and Scott (23) estimated effective sample size using:

$$
n_{\text {deff }}=\bar{n} / \text { deff }
$$

If a data set is $\beta$-binomially distributed, then deff can be defined as (14):

$$
\operatorname{deff}=(1+\bar{n} \theta) /(1+\theta)
$$

The heterogeneity parameter of the $\beta$-binomial distribution, $\theta$, can be estimated from the slope and intercept parameters of the binary power law (14):

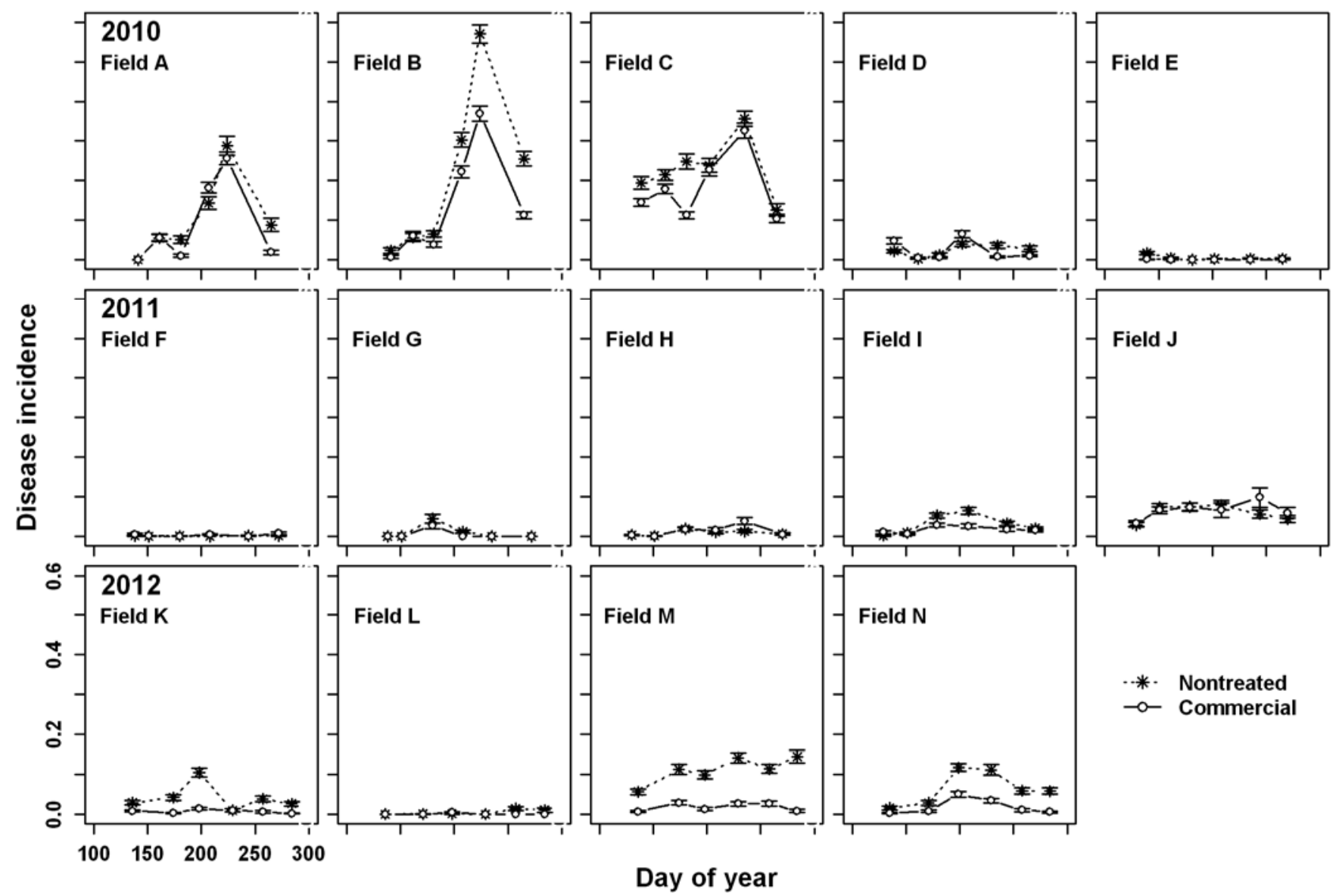

Fig. 1. Temporal progression of Sclerotinia crown rot incidence across 14 pyrethrum fields in northern Tasmania, Australia, from 3 years (2010, 2011, and 2012). Error bars represent the standard error of the mean. 


$$
\theta=\frac{A_{p} \bar{n}-[\hat{p}(1-\hat{p})]^{1-b} / \bar{n}}{[\hat{p}(1-\hat{p})]^{1-b}-A_{p} n^{-b}}
$$

As an alternative, Madden and Hughes (14) developed an estimate of effective sample size for $\beta$-binomially distributed data using an iterative and graphic method, whereby:

$$
n_{v}=\frac{\bar{n}}{\left(0.98135+0.8179 \bar{\theta}+0.004958 \bar{n}+0.30387 \hat{\theta} \bar{n}-0.3471 \hat{\theta}^{2}-0.08475 \hat{\theta}^{2} \bar{n}\right)}
$$

To determine which estimate of $n$ was most appropriate for this data set, a generalized linear modeling approach, similar to that described by Turechek and Madden (28) was employed. Briefly, equation 4 was reformulated into a linear form by completing a complementary $\log -\log$ transformation $[\operatorname{CLL}(z)=\ln (-\ln [1-z])]$ :

$$
\operatorname{CLL}\left(\tilde{p}_{s u}\right)=\ln (n)+\operatorname{CLL}(\hat{p})
$$

with slope $=1$, and the intercept given by the natural log of sample size. Generalized linear modeling in $R$ was used to fit the above equation to obtained data with a known intercept, $\ln (n)$, estimated using $\bar{n}, n_{\text {deff }}$, or $n_{v}$, respectively. A fourth predictor of $n, n_{C L L}$, was obtained by treating equation 9 as a model for the relationship for $\operatorname{CLL}\left(\tilde{p}_{s u}\right): \operatorname{CLL}(\hat{p})$ with an assumed slope of 1 and an unknown intercept. Modeling this relationship as above provided an estimate of $n_{C L L}$ which, in this case, was a parameter describing the mathematical relationship $\operatorname{CLL}\left(\tilde{p}_{s u}\right): \operatorname{CLL}(\hat{p})$ and not necessarily a measure of effective sample size (28). Deviances obtained from the individual model fits was used to compare the predictive power of each estimate of $n$.

Validation of relationship between $\widetilde{\boldsymbol{p}}_{s u}$ and $\widehat{\boldsymbol{p}}$. Data obtained from the additional 72 fields assessed (validation data set) were used to validate predictors of $n$ without recalculation of the $\beta$-binomial or mean sample size parameter estimates. Using equation 9 , each of the four predictors of $n$ obtained with parameters calculated using the model construction data set were used to model the relationship between $\hat{p}$ and $\tilde{p}_{s u}$ in the validation data set. Deviances obtained from generalized linear modeling were used to compare the fit using each predictor of $n$.

The error associated with classifying estimates of $\tilde{p}$ from observed $\tilde{p}_{s u}$ relative to observed $\hat{p}$ were examined for each predictor of $n$. Using values of $p$ ( $\left.p_{\text {threshold }}\right)$ ranging from 0.01 to 0.1 , at 0.01 increments, a series of $\tilde{p}_{s u}$ threshold values $\left(p_{\text {su.threshold }}\right)$ were calculated using equation 4 . For each value of $p_{\text {su.threshold, }}$, observations from the validation sites were then classified as either observed $\hat{p}_{s u}$ less than or greater than $p_{\text {su.threshold. }}$ If observed $\hat{p}_{s u}$ was greater than $p_{\text {su.threshold }}$, it was assumed that $\hat{p}$ was greater than $p_{\text {su.threshold }}$, and vice versa. This assumption was tested by comparing observed $\hat{p}$ to $p_{\text {su.threshold }}$ and calculating the type I ( $\hat{p}$ falsely assumed greater than $\left.p_{\text {su.threshold }}\right)$ and type II ( $\hat{p}$ falsely assumed less than $\left.p_{\text {su.threshold }}\right)$ error rates. This was repeated for each predictor of $n$. Calculations were performed in $R$.

\section{Results}

Disease incidence. Diseased plants were observed in all plots but not at all sampling periods. In the commercial plot of two fields, one each in 2011 (field G) and 2012 (field L), SCR was only observed during a single sampling period (Fig. 1). Of a total of 168 plot assessments (14 fields, two plots per field, and six assessments each), SCR was not observed on 27 occasions. Of these, 17 instances of zero disease were observed in commercial plots and 10 in nontreated plots, constituting 20.2 and $11.9 \%$ of these plot assessments,

\begin{tabular}{|c|c|c|c|c|c|c|c|}
\hline Year, field & Plot $^{\mathrm{a}}$ & Model of best fit ${ }^{b}$ & $P$ & Slope & Intercept & $\boldsymbol{R}^{* 2 \mathrm{c}}$ & $\mathbf{R S E}^{\mathbf{d}}$ \\
\hline \multicolumn{8}{|l|}{2010} \\
\hline \multirow[t]{2}{*}{ Field A } & $\mathrm{N}$ & Monomolecular & 0.53 & 0.001 & -0.179 & 0.33 & 0.11 \\
\hline & $\mathrm{C}$ & Monomolecular & 0.77 & 0.002 & -0.085 & 0.12 & 0.13 \\
\hline \multirow[t]{2}{*}{ Field B } & $\mathrm{N}$ & Monomolecular & 0.45 & 0.004 & -0.745 & 0.50 & 0.28 \\
\hline & $\mathrm{C}$ & Monomolecular & 0.54 & 0.002 & -0.268 & 0.33 & 0.16 \\
\hline \multirow[t]{2}{*}{ Field C } & $\mathrm{N}$ & Exponential & 0.99 & -0.001 & 0.276 & 0.00 & 0.38 \\
\hline & $\mathrm{C}$ & Monomolecular & 0.91 & 0.001 & 0.102 & 0.04 & 0.12 \\
\hline \multirow[t]{2}{*}{ Field D } & $\mathrm{N}$ & Monomolecular & 0.49 & 0.000 & -0.011 & 0.32 & 0.01 \\
\hline & $\mathrm{C}$ & Monomolecular & 0.83 & -0.000 & 0.055 & 0.08 & 0.03 \\
\hline \multirow[t]{2}{*}{ Field E } & $\mathrm{N}$ & Linear & 0.47 & -0.000 & 0.020 & 0.34 & 0.01 \\
\hline & $\mathrm{C}$ & Logistic & 0.93 & -0.038 & 0.001 & 0.33 & 0.78 \\
\hline \multicolumn{8}{|l|}{2011} \\
\hline \multirow[t]{2}{*}{ Field F } & $\mathrm{N}$ & Monomolecular & 0.57 & 0.000 & -0.000 & 0.24 & 0.00 \\
\hline & $\mathrm{C}$ & Monomolecular & 0.84 & 0.000 & -0.000 & 0.08 & 0.00 \\
\hline \multirow[t]{2}{*}{ Field G } & $\mathrm{N}$ & Monomolecular & 0.79 & -0.000 & 0.019 & 0.02 & 0.02 \\
\hline & $\mathrm{C}$ & Monomolecular & 0.75 & -0.000 & 0.012 & 0.03 & 0.01 \\
\hline \multirow[t]{2}{*}{ Field H } & $\mathrm{N}$ & Monomolecular & 0.59 & 0.000 & 0.002 & 0.08 & 0.01 \\
\hline & $\mathrm{C}$ & Monomolecular & 0.38 & 0.000 & -0.010 & 0.20 & 0.01 \\
\hline \multirow[t]{2}{*}{ Field I } & $\mathrm{N}$ & Monomolecular & 0.51 & 0.000 & -0.000 & 0.12 & 0.03 \\
\hline & $\mathrm{C}$ & Monomolecular & 0.57 & 0.000 & 0.008 & 0.09 & 0.01 \\
\hline \multirow[t]{2}{*}{ Field J } & $\mathrm{N}$ & Monomolecular & 0.98 & 0.000 & 0.058 & 0.00 & 0.02 \\
\hline & $\mathrm{C}$ & Monomolecular & 0.27 & 0.000 & 0.024 & 0.30 & 0.02 \\
\hline \multicolumn{8}{|l|}{2012} \\
\hline \multirow[t]{2}{*}{ Field K } & $\mathrm{N}$ & Monomolecular & 0.71 & -0.000 & 0.067 & 0.04 & 0.04 \\
\hline & $\mathrm{C}$ & Monomolecular & 0.48 & -0.000 & 0.013 & 0.14 & 0.00 \\
\hline \multirow[t]{2}{*}{ Field L } & $\mathrm{N}$ & Linear & 0.07 & 0.000 & -0.014 & 0.60 & 0.00 \\
\hline & $\mathrm{C}$ & Monomolecular & 0.81 & -0.000 & 0.002 & 0.02 & 0.00 \\
\hline \multirow[t]{2}{*}{ Field M } & $\mathrm{N}$ & Monomolecular & 0.04 & 0.001 & 0.003 & 0.69 & 0.02 \\
\hline & $\mathrm{C}$ & Monomolecular & 0.82 & 0.000 & 0.013 & 0.02 & 0.01 \\
\hline \multirow[t]{2}{*}{ Field N } & $\mathrm{N}$ & Logistic & 0.20 & 0.009 & 0.008 & 0.08 & 0.74 \\
\hline & $\mathrm{C}$ & Linear & 0.94 & 0.000 & 0.015 & 0.00 & 0.02 \\
\hline
\end{tabular}
respectively. Mean disease incidence across all plots and time

Table 1. Temporal disease progress models of best fit defining the incidence of Sclerotinia crown rot in 14 pyrethrum fields in Tasmania, Australia

${ }^{a}$ One nontreated $(\mathrm{N})$ plot and one commercially managed plot $(\mathrm{C})$ were established in each field. Disease assessments were undertaken from austral midautumn (April to May) to austral mid-spring (September to October) each year, with all crops assessed six times.

${ }^{b}$ Model of best fit selected by fitting the linearized forms of the linear, monomolecular, exponential, logistic, and Gompertz models. Selection was based on comparison of the $F$ statistics for linearity, with further evidence gained by back transforming predicted values of each model, fitting these to the observed values and comparing coefficients of determination.

${ }^{\mathrm{c}}$ Coefficient of variation of the regression between the back-transformed predicted values of the model of best fit and observed values.

${ }^{\mathrm{d}}$ Residual standard error of back-transformed regression. 

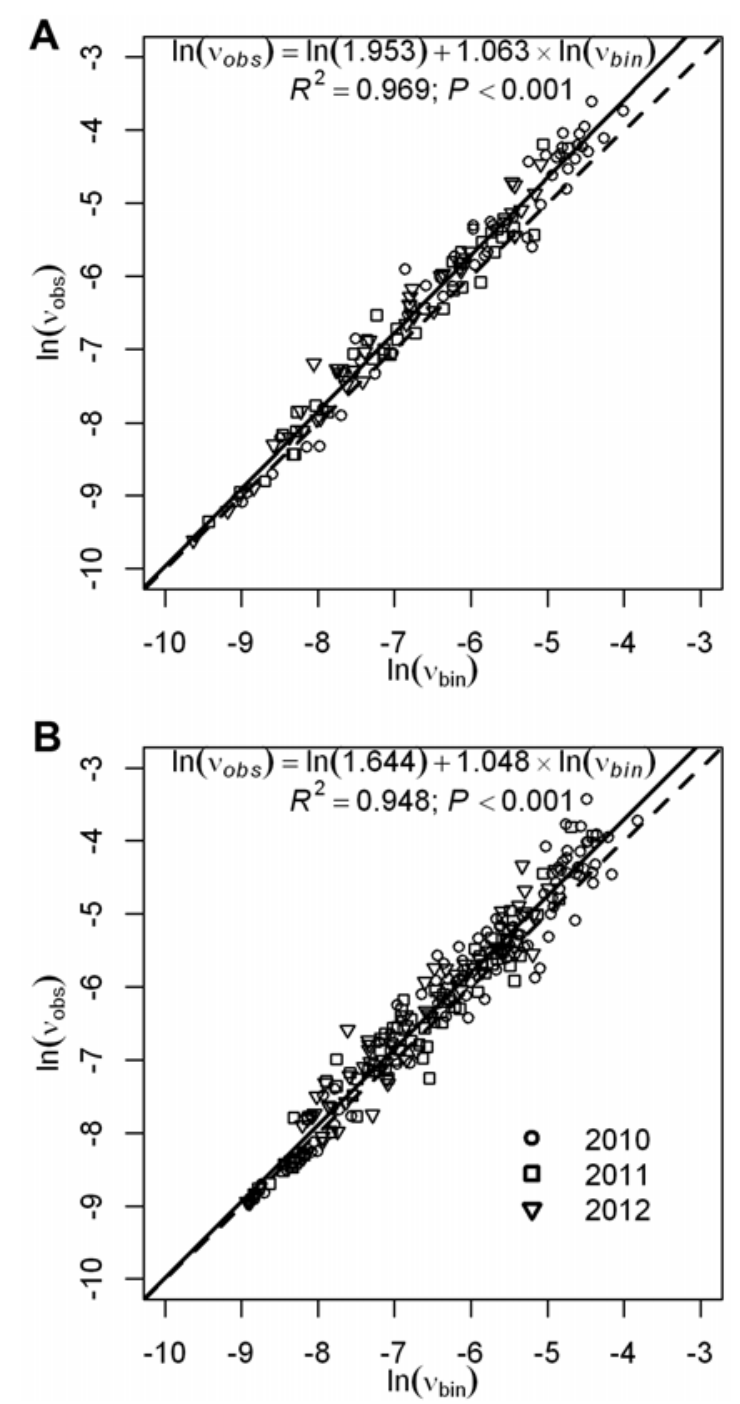

Fig. 2. Relationship between the natural logs of observed variance $\left(v_{o b s}\right)$ and theoretical binomial variance $\left(v_{\text {bin }}\right)$ of Sclerotinia crown rot incidence in pyrethrum fields from Tasmania, Australia. Plots represent variance relationships at the scale of A, plot and B, transects within a plot. Solid line represents the fitted model described by the equation given in each plot. Dashed line represents the theoretical relationship under a binomial (random) distribution of disease. periods was 0.053 (range 0 to 0.57 ). Greater mean disease incidence was observed in nontreated plots $(0.065$, range 0 to 0.57$)$, relative to commercial plots $(0.041$, range 0 to 0.37$)$. Within each year, mean disease incidence was 0.100 (range 0 to 0.57 ) in 2010, 0.021 (range 0 to 0.098 ) in 2011 , and 0.033 (range 0 to 0.14 ) in 2012.

Temporal analysis. The standard disease progress models poorly described disease incidence in most cases (Table 1). Only two instances of adequate fit were observed: the nontreated plots at sites field M (monomolecular) and field L (linear), where the fitted models accounted for 69 and $60 \%$ of observed incidence, respectively. In all other instances, the coefficient of determination was less than $35 \%$ for the model of best fit.

Binary power law. Model construction data set. The binary power law provided a good fit to the data at the scales of both the plots and individual transects within plots, with coefficients of determination $\left(R^{2}\right)$ of greater than 0.94 (Fig. 2). At the plot and transect scale, calculated slope parameters were 1.063 and 1.048, respectively. Both estimates were greater than $1(P<0.0001)$, whereas differences in estimates between scales were not significant $(P=0.339)$. Estimated intercept parameters were 0.669 and 0.497 at the plot and transect scale, respectively, and did not differ significantly $(P=0.100)$. Both estimates of the intercept parameter were significantly greater than $0(P<0.0001)$. These results indicated that disease incidence was aggregated at both the transect and plot scales, and the degree of aggregation changed systematically with disease incidence.

Covariance analysis indicated that including the factor Year as a covariate had no significant effect on estimate of slope $(P \geq 0.121)$ or intercept $(P \geq 0.112)$ at either the plot or transect scale (Table 2$)$. Therefore, the effects of the factors Treatment, Site, and Sample period on slope and intercept were recalculated across the whole data set rather than within individual years. Sequentially adding each of these factors individually to the slope and intercept did not significantly alter parameter estimates at either spatial scale $(P \geq$ 0.059 ; Table 2).

Model validation data set. The binary power law provided a good fit to the validation data set, with $R^{2}$ of 0.95 . Calculated slope (1.081) and intercept $(0.837)$ parameters were significantly greater than 1 and $0(P<0.001)$, respectively. Neither parameter estimate differed significantly from those obtained at the plot scale for the model data set $(P=0.233$ and $P=0.112)$.

Correlation-based spatial analyses. Spatial autocorrelation. Significant first-order spatial autocorrelation, $\hat{r}_{1}$, was detected in $19.4 \%$ of all transects $(P \leq 0.05)$. The frequency distribution of $\hat{r}_{1}$ across the entire data set was -0.52 to 0.96 , with mean 0.105 and

Table 2. Covariance analysis of the effect of Treatment, Site, Sampling period, and Year across all data, on the slope $\left(\ln \left[A_{p}\right]\right)$ and intercept $(b)$ parameters of the binary power law for the incidence of Sclerotinia crown rot in pyrethrum fields in Tasmania, Australia ${ }^{a}$

\begin{tabular}{|c|c|c|c|c|c|c|c|c|c|c|}
\hline \multirow[b]{2}{*}{ Model $^{b}$} & \multirow[b]{2}{*}{$d f_{S S E^{\mathrm{c}}}^{\mathrm{c}}$} & \multirow[b]{2}{*}{$d f_{\text {factor }}{ }^{\mathrm{d}}$} & \multicolumn{4}{|c|}{$\ln \left(A_{p}\right)$} & \multicolumn{4}{|c|}{ b } \\
\hline & & & SSE & Diff & $F$ & $P$ & SSE & Diff & $F$ & $P$ \\
\hline \multicolumn{11}{|l|}{ Plot scale } \\
\hline Binary power law & 128 & $\ldots$ & 8.593 & $\ldots$ & $\cdots$ & $\ldots$ & 8.593 & . & $\cdots$ & $\ldots$ \\
\hline+ Year & 126 & 2 & 8.300 & 0.293 & 2.225 & 0.112 & 8.310 & 0.283 & 2.150 & 0.121 \\
\hline +Treatment & 127 & 1 & 8.456 & 0.137 & 2.065 & 0.153 & 8.334 & 0.239 & 3.635 & 0.059 \\
\hline+ Site & 127 & 1 & 8.556 & 0.037 & 0.552 & 0.459 & 8.558 & 0.035 & 0.515 & 0.474 \\
\hline +Sample period & 127 & 1 & 8.444 & 0.149 & 2.247 & 0.136 & 8.467 & 0.126 & 1.893 & 0.171 \\
\hline \multicolumn{11}{|l|}{ Transect scale } \\
\hline Binary power law & 241 & $\ldots$ & 23.492 & . & $\ldots$ & $\ldots$ & 23.492 & & $\ldots$ & $\ldots$ \\
\hline +Year & 239 & 2 & 23.264 & 0.229 & 1.175 & 0.311 & 23.236 & 0.256 & 1.315 & 0.271 \\
\hline +Treatment & 240 & 1 & 23.365 & 0.127 & 1.304 & 0.255 & 23.272 & 0.220 & 2.271 & 0.133 \\
\hline+ Site & 240 & 1 & 23.489 & 0.003 & 0.029 & 0.864 & 23.481 & 0.011 & 0.116 & 0.734 \\
\hline +Sample period & 240 & 1 & 23.490 & 0.002 & 0.018 & 0.893 & 23.488 & 0.004 & 0.042 & 0.838 \\
\hline
\end{tabular}

${ }^{a}$ SSE $=$ sum of squares error; Diff $=$ difference in SSE between the binary power law model and the alternative, a binary power law model with relevant factor included as a covariate in either the slope or intercept term; $F=F$ test for significance of the difference in SSE between models, where $F=$ (factor $\left.\mathrm{SSE} / d f_{\text {factor }}\right) /\left(\right.$ model SSE$\left./ d f_{S S E}\right)$; and $P$ represents the probability of significance of this test.

${ }^{b}$ Disease assessments were undertaken on one nontreated plot and one commercially managed plot in each field with all crops assessed six times. At each sampling time point, disease was measured within each plot along two $25-\mathrm{m}$-long transects, with sampling units at each 1 -m interval $(N=25)$. The binary power law was fit to data from both transects (plot scale) or to data from each transect separately (transect scale).

${ }^{c}$ Degrees of freedom for the binary power law model SSE.

${ }^{\mathrm{d}}$ Degrees of freedom for factor included as a covariate of either the slope or intercept term within the binary power law model. 
median 0.089. Within treatments, $\hat{r}_{1}$ estimates from nontreated and commercial plots displayed similar distribution patterns (Fig. 3B and D), with means which did not differ significantly $(P=0.146)$. First-order spatial autocorrelation tended to increase with disease incidence (Fig. 4). Spatial autocorrelation decreased at the second order, where mean $\hat{r}_{2}$ was 0.033 and median $\hat{r}_{2}$ 0.017. Of the 51 transects where significant first-order spatial autocorrelation was detected, 13 also had significant second-order spatial autocorrelation. Additionally, of these 13, 3 and 2 had significant third- and fourth-order autocorrelation, respectively.

SADIE. Spatial aggregation, as indicated by the index of aggregation significantly greater than $1(P \leq 0.025$, two-tailed test), was detected in $16.8 \%$ of all transects. In all, 23 of 127 nontreated transects $(18.1 \%)$ were significantly aggregated, as were 18 of 116 commercial transects (15.5\%). Across years, 34 of 96 (35.4\%) transects had SCR that was significantly aggregated in 2010, whereas only 3 of $75(4.0 \%)$ and 5 of $72(6.9 \%)$ transects had significant aggregation in 2011 and 2012, respectively. Within the 41 transects, the average number of red clusters detected was 3.05 (range 1 to 6). Average size of red clusters was 2.30 sampling units (range 1 to 10).
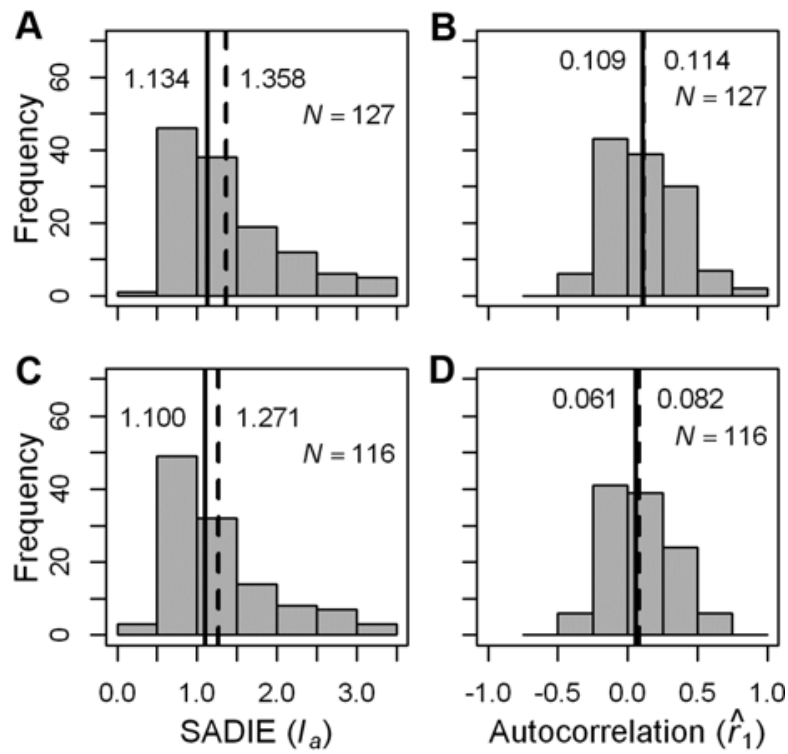

Fig. 3. Frequency distributions of $\mathbf{A}$ and $\mathbf{C}$, first-order spatial autocorrelation coefficients and $\mathbf{B}$ and $\mathbf{D}$, spatial analysis by distance indices (SADIE) indices of aggregation of Sclerotinia crown rot incidence in pyrethrum fields in Tasmania, Australia. Disease assessments were conducted in a total of 14 commercial pyrethrum fields from 2010 to 2012. Fields contained plots that either received no fungicide applications for disease control (A and B), or applications according to commercial recommendations ( $\mathbf{C}$ and $\mathbf{D})$. Solid vertical lines indicate the median values while dashed lines indicate the mean.
Mean index of aggregation for all transects analyzed was 1.315 (range 0.418 to 3.492 ). Mean $I_{a}$ was not significantly different $(P=$ 0.313 ) between nontreated plots and commercial plots (Fig. 3A and C). However, mean $I_{a}$ in 2010 (1.646) was greater than both $2011(1.080)$ and $2012(1.120)(P<0.0001)$ but not different between 2011 and $2012(P=0.66)$. Although values of $I_{a}$ are not directly proportional to degrees of significance, all transects that were significantly aggregated had $I_{a}$ values of 2.01 or greater.

Relationships between disease incidence at the sampling unit and plant scales. Using $\bar{n}$ and, thus, assuming a binomial distribution provided the poorest prediction of the relationship between $p$ and $p_{s u}$ (Table 3; Fig. 5A). Of the two predictors of $n$ that accounted for spatial heterogeneity, $n_{\text {deff }}$ produced a smaller model deviance than $n_{v}$. The smallest model deviance was obtained when modeling the complementary log-logs of $p$ and $p_{s u}$ with no assumed intercept parameter $\left(n_{C L L}\right)$. Relative to the calculated value of $n_{C L L}(17.0), n_{\text {deff }}$ (15.7) and $n_{v}$ (18.9) underestimated and overestimated, respectively, the effective sample size in this pathosystem.

Validation of relationship between $\boldsymbol{p}_{s u}$ and $\boldsymbol{p}$. Deviance of the generalized linear model between $\hat{p}$ and $\tilde{p}_{s u}$ was minimized using the effective sample size predictor $n_{\text {deff }}$ (31.30; Table 3; Fig. 5B). Greatest model deviance was observed when $\bar{n}$ was used as the

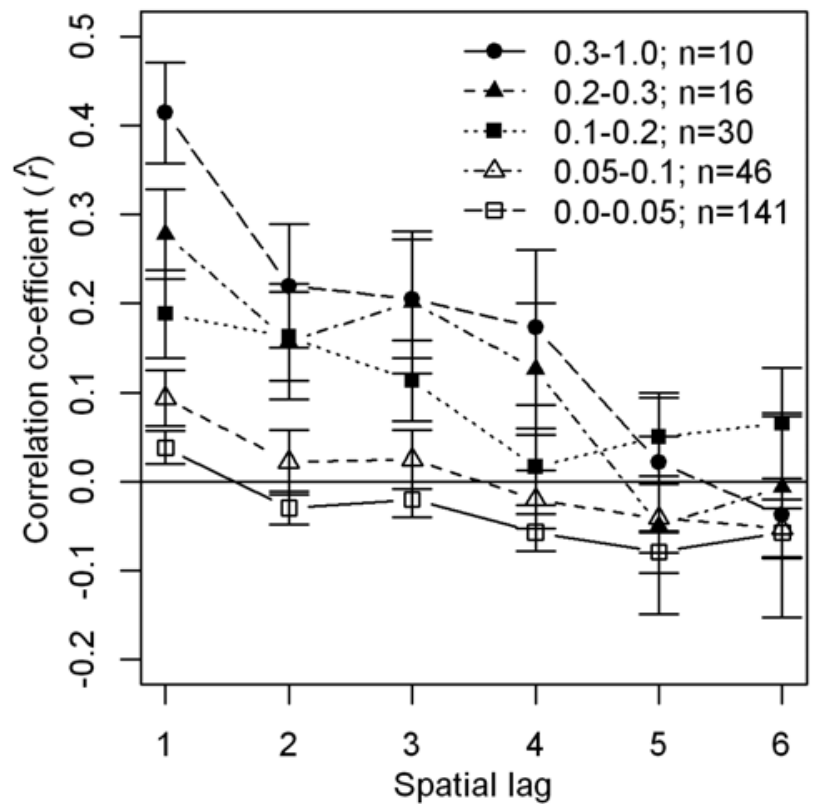

Fig. 4. Change in mean spatial autocorrelation coefficient $(r)$ with spatial lag across five categories of Sclerotinia crown rot incidence. Means are calculated from observations across six sampling periods in 3 years $(2010,2011$, and 2012) from 14 pyrethrum fields in Tasmania, Australia. Errors bars represent the standard error of the mean.

Table 3. Estimates and model fit parameters of four models for an effective sample size, describing the hierarchical relationship between incidence of pyrethrum plants with Sclerotinia crown rot and the incidence of quadrats with at least one pyrethrum plant with Sclerotinia crown rot

\begin{tabular}{|c|c|c|c|c|c|}
\hline \multirow[b]{2}{*}{ Predictor variable $^{c}$} & \multirow[b]{2}{*}{ Value } & \multicolumn{2}{|c|}{ Model data set ${ }^{\mathrm{a}}$} & \multicolumn{2}{|c|}{ Validation data set ${ }^{b}$} \\
\hline & & Deviance & $d f$ & Deviance & $d f$ \\
\hline $\bar{n}$ & 20.75 & 14.61 & 119 & 52.95 & 263 \\
\hline$n_{\text {deff }}$ & 15.67 & 10.73 & 119 & 31.30 & 263 \\
\hline$n_{v}$ & 18.91 & 11.25 & 119 & 41.20 & 263 \\
\hline$n_{C L L}$ & 17.02 & 9.92 & 119 & 33.35 & 263 \\
\hline
\end{tabular}

${ }^{a}$ Fit of modeled relationship to the modeled data set incorporating data collected from 14 pyrethrum fields spread across three years (2010, 2011, and 2012) and scored at six time points within a given year. In total, 50 sampling units were collected from each of two plots (one nontreated and one commercially managed plot); $d f=$ degrees of freedom.

${ }^{\mathrm{b}}$ Fit of modeled relationship to the validation data set incorporating data collected from 72 pyrethrum fields spread across three years (2010, 2011, and 2012) and scored at three time points within a given year. In total, 25 sampling units were collected from each of two plots (one nontreated and one commercially managed plot); $d f=$ degrees of freedom.

${ }^{c}$ Estimates of effective sample size: $\bar{n}$, the mean number of plants within a sampling unit; $n_{\text {deff }}$, the effective sample size accounting for spatial heterogeneity according to Rao and Scott (23); $n_{v}$, the effective sample size accounting for spatial heterogeneity according to Madden and Hughes (14); and $n_{C L L}$, the descriptor of the relationship between the complementary log-logs of disease incidence at the plant and sampling unit scales. 
predictor of sample size (52.95). Type I errors (false positives) were minimized when sample size was predicted by $\bar{n}$ across threshold incidences from 0.01 to 0.10 , while type I errors were maximized when using $n_{\text {deff }}$ (Fig. 6A). Conversely, type II errors (false negatives) were minimized when $n_{\text {deff }}$ was used, whereas $\bar{n}$ maximized type II errors across all threshold incidences tested (Fig. 6B)

\section{Discussion}

The temporal and spatial heterogeneity of SCR incidence in Australian pyrethrum fields was characterized in this study. Disease incidence was generally low regardless of location, with disease incidence less than $10 \%$ in 139 of 168 observations $(82.7 \%)$.
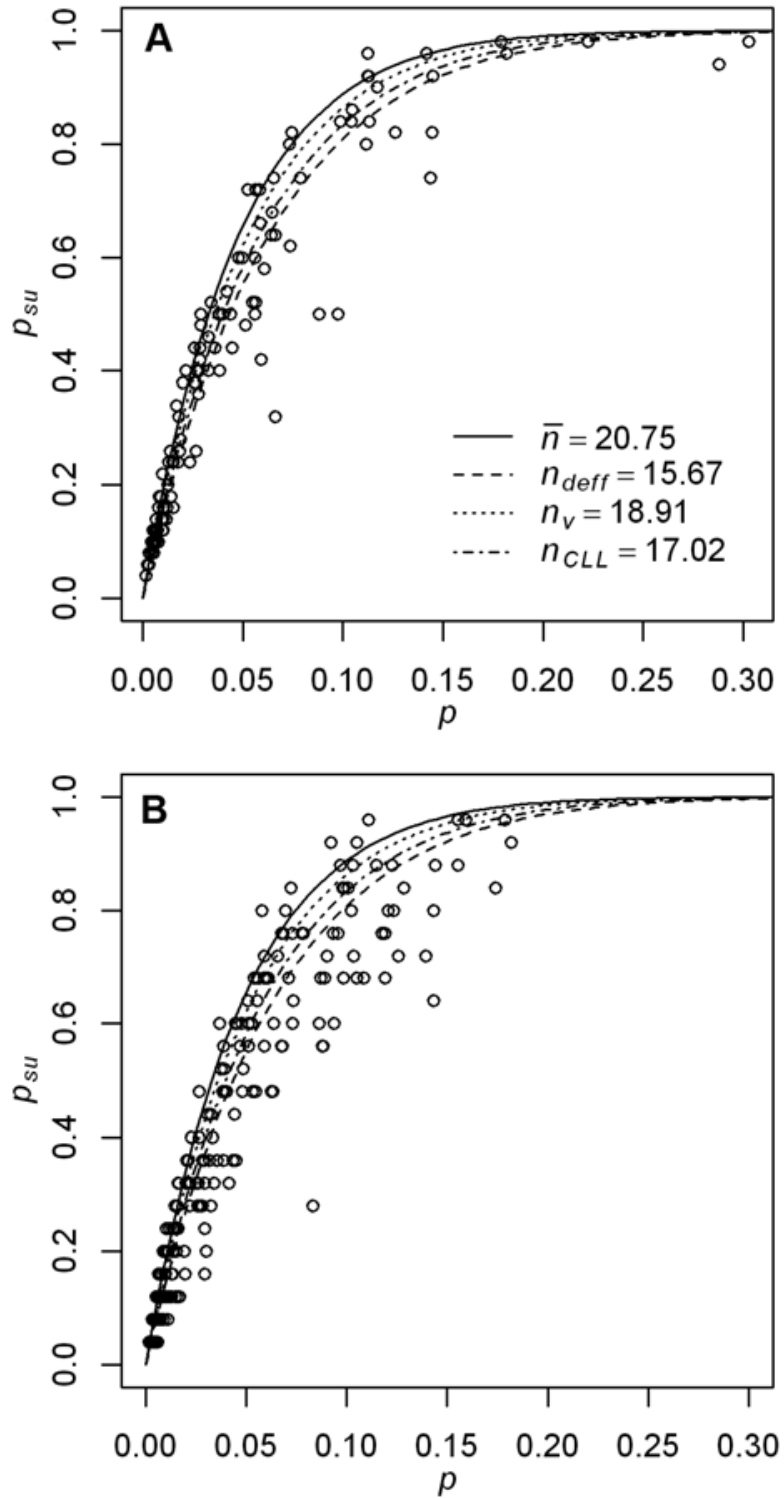

Fig. 5. Relationship between the incidence of pyrethrum plants with Sclerotinia crown rot $(p)$ and incidence of quadrats (sampling unit $\left[p_{s u}\right]$ ) where at least one plant had Sclerotinia crown rot. A, Initially, modeling was conducted using data collected from 14 pyrethrum fields spread across 3 years (2010, 2011, and 2012) and scored at six time points within a given year. B, Estimated curves were then compared with data from 72 pyrethrum fields scored at three time points within a year, spread across the same 3 years. Shape of the modeled curve was influenced by the estimate of the effective sample size (provided numerically in figures), with four options explored: $\bar{n}$, the mean number of plants within a sampling unit; $n_{\text {deff, }}$ the effective sample size accounting for spatial heterogeneity according to Rao and Scott (23); $n_{v}$, the effective sample size accounting for spatial heterogeneity according to Madden and Hughes (14); and $n_{C L L}$, the descriptor of the relationship between the complementary log-logs of disease incidence at the plant and sampling unit scales.
Disease incidence was greater than $20 \%$ in only 13 observations $(7.7 \%)$, with a maximum disease incidence of $57 \%$ observed. Temporally, disease incidence was typically observed to increase during the autumn and winter sampling periods but then decrease during spring. The timing of these observations may be explained by the developmental physiology of the host (21). During autumn and winter, pyrethrum enters a semidormant state as small prostrate bushes, the leaves of which may contact the soil surface. Infections via myceliogenic germination of sclerotia of Sclerotinia spp. require close proximity to host tissue $(7,11)$. Field observations during this study suggest that infection via leaf contact with the soil is common. However, with the onset of spring, pyrethrum crops undergo renewed vegetative growth and assume a more upright habit, which reduces leaf contact with the soil surface. Thus, as the plant matures, the combination of reduced host density due to plant death from SCR throughout autumn and winter and reduced soil contact could reduce the opportunity for new infections during early spring. Ascosporic infection is thought to have a minimal, if any, role in SCR (21). Apothecial formation by $S$. minor is rarely observed in the field $(3,4,6,22)$ and, although $S$. sclerotiorum is able to more readily produce ascospores, observations and dedicated surveys have indicated that this is a rare occurrence in pyrethrum fields over the winter and early spring period (22).

Spatial analyses indicated that disease was aggregated primarily at the scale of the sampling unit or below, and the extent of spatial aggregation at greater spatial scales was limited. The binary power law indicated aggregation at the sampling unit scale and below and that the degree of this aggregation was influenced by disease incidence. Correlation-based analyses supported these results. Results from SADIE indicated that, in transects in which significant spatial aggregation was present, the average length of the aggregation was 2.3 sampling units, where each sampling unit was $1 \mathrm{~m}$ long. This is in agreement with the results of spatial autocorrelation analyses, where the median lag distance over which spatial autocorrelation was detected was 1; thus, only between two neighboring sampling units. Mean first-order spatial autocorrelation coefficients increased with disease incidence. Overall, the aggregation of SCR is generally similar to that observed for Sclerotinia flower blight of pyrethrum, which indicated that spatial aggregation of disease incidence occurs primarily at the scale of 1 to $2 \mathrm{~m}$ (22). For SCR, these findings further support the hypothesis that infection of the
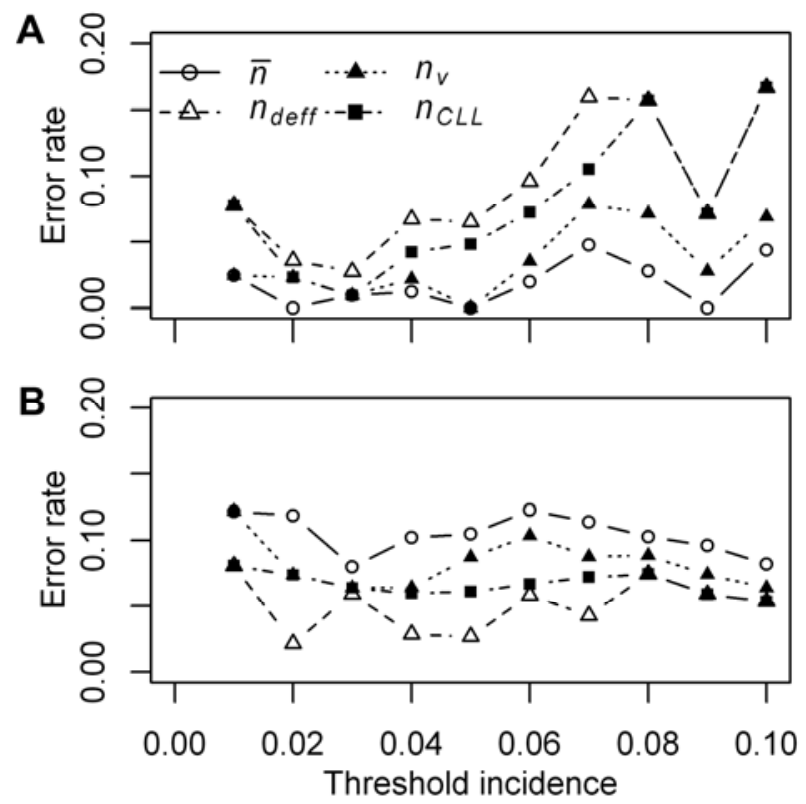

Fig. 6. Error rate of individual estimates of effective sample size against various thresholds of plants with Sclerotinia crown rot (SCR). A, Type I error rate, the proportion of false predictions of SCR incidence greater than or equal to threshold incidence; and $\mathbf{B}$, type II error, the proportion of false predictions of the incidence of pyrethrum plants with Sclerotinia crown rot less than the threshold incidence. 
vegetative tissues of pyrethrum by Sclerotinia spp. is via localized spread from soil to plant and between neighboring plants. Such aggregation has also been described in other crops, notably lettuce $(6,7,11)$. Hao and Subbarao (7) noted a strong correlation in the spatial patterns of sclerotial inoculum and lettuce drop. In lettuce, practices that reduced inoculum density in the soil reduced disease incidence and delayed the onset of spatial aggregation (7). Similar strategies may also be efficacious for SCR management in pyrethrum, as may methods to minimize plant contact with inoculum and reduce canopy density.

The industry currently employs an SCR control threshold of $2 \%$ diseased plants, above which fungicide applications are applied. For management purposes, obtaining a precise and accurate estimate of disease incidence is not always practical or efficient. Thus, alternatives such as hierarchical sampling where the incidence of diseased sampling units are counted may be beneficial $(8,10)$. Although this constitutes a decreased level of accuracy of disease estimates, as observed in our results, the increased efficiency gains through speed and ease of scoring may outweigh loss of precision. In this study, the predictors of effective sample size that accounted for spatial variability produced an improved model fit over the mean sample size, which inherently assumes a binomial distribution. Although the predictor $n_{C L L}$ produced the best fit to the modeling data set, it was outperformed by Rao and Scott's (23) predictor of effective sample size $\left(n_{\text {deff }}\right)$ when using independent observations for validation. Given this, and the fact that $n_{C L L}$ has no inherent biological assumptions in its derivation (29), it is believed that $n_{\text {deff }}$ provides the more robust predictor of effective sample size in this pathosystem. However, the selection of a value of effective sample size is not necessarily clear-cut and may be dependent upon the costs associated with incorrect management decisions. Under a risk-averse strategy that seeks to minimize crop damage due to false-negative classification errors, the hierarchical model based on $n_{\text {deff }}$ as the effective sample size parameter would be the preferred predictor over most threshold values of disease incidence. Additionally, $n_{\text {deff }}$ also produced the smallest deviance, when compared with the validation data set, which suggests that it would also be appropriate in situations when minimizing overall misclassification errors is desired. Conversely, if the cost of false-positive classifications is higher than for false-negative classifications, $\bar{n}$ could be accepted as the effective sample size. Although this would increase the overall rate of error, it would minimize the instances of falsely predicting disease incidences greater than the control threshold.

Acceptance of a given hierarchical relationship for use in disease management decision-making depends on the efficacy of the commercial management program, yield loss associated with SCR, and their relative costs. In a study of yield damage from SCR and efficacy of the commercial management program, grower management systems did reduce the incidence of the disease, on average, 43 to $67 \%$ compared with nontreated plots (27). However, there was not an overall improvement in flower yield across all sites. This suggests that reevaluation of provisional disease thresholds is warranted, and may require consideration of other risk factors of disease outbreaks beyond a simple threshold of the incidence of affected plants. Nonetheless, the results presented here suggest that management practices that reduce the initial sclerotial load in the soil may provide significant improved control of the disease. Further work to test this hypothesis is needed. In the interim, the improved understanding of the relationship between disease incidence at different scales of measurement provides a foundation for development of improved sampling approaches, whether these are hierarchical or based on other sampling methods (15).

\section{Acknowledgments}

Funding for this research was provided by the Botanical Resources AustraliaAgricultural Services Pty Ltd. (BRA), the United States Department of Agriculture-Agricultural Research Service CRIS 5358-21000-035-00, and the Australian Research Council (ARC) as part of the ARC-Linkage program (project LP100100529). We thank C. Palmer (Tasmanian Institute of Agriculture) for excellent technical assistance during the course of this study, G. Hughes (Scotland's Rural College) and M. Twomey (Oregon State University) for their review and helpful suggestions, and the field staff and growers of BRA for providing access to commercial fields.

\section{Literature Cited}

1. Adams, P. B., and Ayers, W. A. 1979. Ecology of Sclerotinia species. Phytopathology 69:896-899.

2. Bolton, M. D., Thomma, B., and Nelson, B. D. 2006. Sclerotinia sclerotiorum (Lib.) de Bary: biology and molecular traits of a cosmopolitan pathogen. Mol. Plant Pathol. 7:1-16.

3. Dillard, H. R., and Grogan, R. G. 1985. Relationship between sclerotial spatial pattern and density of Sclerotinia minor and the incidence of lettuce drop. Phytopathology 75:90-94.

4. Ekins, M. G., Aitken, E. A. B., and Goulter, K. C. 2002. Carpogenic germination of Sclerotinia minor and potential distribution in Australia. Australas. Plant Pathol. 31:259-265.

5. Elliott, M. 1995. Chemicals in insect control. Pages 3-31 in: Pyrethrum Flowers: Chemistry, Toxicology and Uses. J. E. Casida and G. B. Quistad, eds. Oxford University Press, New York.

6. Hao, J. J., and Subbarao, K. V. 2005. Comparative analyses of lettuce drop epidemics caused by Sclerotinia minor and S. sclerotiorum. Plant Dis. 89:717-725.

7. Hao, J. J., and Subbarao, K. V. 2006. Dynamics of lettuce drop incidence and Sclerotinia minor inoculum under varied crop rotations. Plant Dis. 90:269-278.

8. Hughes, G., Gottwald, T. R., and Levy, L. 2002. The use of hierarchical sampling in the surveillance program for Plum pox virus incidence in the United States. Plant Dis. 86:259-263.

9. Hughes, G., and Madden, L. V. 1992. Aggregation and incidence of disease Plant Pathol. 41:657-660.

10. Hughes, G., McRoberts, N., Madden, L. V., and Gottwald, T. R. 1997. Relationships between disease incidence at two levels in a spatial hierarchy. Phytopathology 87:542-550.

11. Isnaini, M., and Keane, P. J. 2007. Biocontrol and epidemiology of lettuce drop caused by Sclerotinia minor at Bacchus Marsh, Victoria. Australas. Plant Pathol. 36:295-304.

12. Jones, S. J., Gent, D. H., Pethybridge, S. J., and Hay, F. S. 2012. Site-specific risk factors of white mould epidemics in bean (Phaseolus vulgaris) in Tasmania, Australia. N. Z. J. Crop Hortic. Sci. 40:147-159.

13. Macdonald, W. L. 1995. Pyrethrum flowers-production in Australia. Pages 55-66 in: Pyrethrum Flowers: Chemistry, Toxicology and Uses. J. E. Casida and G. B. Quistad, eds. Oxford University Press, New York.

14. Madden, L. V., and Hughes, G. 1999. An effective sample size for predicting plant disease incidence in a spatial hierarchy. Phytopathology 89:770-781.

15. Madden, L. V., Hughes, G., and van den Bosch, F. 2007. The Study of Plant Disease Epidemics. American Phytopathological Society, St. Paul, MN.

16. Neher, D. A., Reynolds, R. L., and Campbell, C. L. 1997. Analysis of disease progress curves using linear models. Pages 29-33 in: Exercises in Plant Disease Epidemiology. L. J. Francl and D. A. Neher, eds. American Phytopathological Society, St. Paul, MN.

17. Nutter, F. W. 1997. Quantifying the temporal dynamics of plant virus epidemics: a review. Crop Prot. 16:603-618.

18. Perry, J. N. 1995. Spatial analysis by distance indices. J. Anim. Ecol 64:303-314.

19. Perry, J. N. 1998. Measures of spatial pattern for counts. Ecology 79:10081017.

20. Perry, J. N., Winder, L., Holland, J. M., and Alston, R. D. 1999. Red-blue plots for detecting clusters in count data. Ecol. Lett. 2:106-113.

21. Pethybridge, S. J., Hay, F. S., Esker, P. D., Gent, D. H., Wilson, C. R., Groom, T., and Nutter, F. W. 2008. Diseases of pyrethrum in Tasmania: challenges and prospects for management. Plant Dis. 92:1260-1272.

22. Pethybridge, S. J., Hay, F. S., and Gent, D. H. 2010. Characterization of the spatiotemporal attributes of Sclerotinia flower blight epidemics in a perennial pyrethrum pathosystem. Plant Dis. 94:1305-1313.

23. Rao, J. N. K., and Scott, A. J. 1992. A simple method for the analysis of clustered binary data. Biometrics 48:577-585.

24. R Development Core Team. 2009. R: A Language and Environment for Statistical Computing. R Foundation for Statistical Computing, Vienna.

25. Reynolds, K. M., and Madden, L. V. 1988. Analysis of epidemics using spatio-temporal autocorrelation. Phytopathology 78:240-246.

26. Reynolds, K. M., Madden, L. V., and Ellis, M. A. 1988. Spatio-temporal analysis of epidemics development of leather rot of strawberry. Phytopathology 78:246-252.

27. Scott, J. B., Pethybridge, S. J., Gent, D. H., Groom, T., and Hay, F. S. 2014 Crop damage from Sclerotinia crown rot and risk factors in pyrethrum. Plant Dis. 98:103-111.

28. Turechek, W. W., and Madden, L. V. 2003. A generalized linear modeling approach for characterizing disease incidence in a spatial hierarchy. Phytopathology 93:458-466.

29. Turechek, W. W., and Mahaffee, W. F. 2004. Spatial pattern analysis of hop powdery mildew in the Pacific Northwest: Implications for sampling. Phytopathology 94:1116-1128.

30. Zito, S. W., Zieg, R. G., and Staba, E. J. 1983. Distribution of pyrethrins in oil glands and leaf tissue of Chrysanthemum cinerariafolium. Planta Med. 47:205-207. 